\title{
FACTORES QUE INFLUYEN EN EL NIVEL DE SATISFACCIÓN DE LOS TURISTAS EXTRANJEROS QUE VISITAN CUSCO
}

\author{
Carmen Domínguez Díaz ${ }^{1}$ \& Olga Solano Dávila ${ }^{2}$
}

Resumen.- En el presente trabajo se determinaron los factores más relevantes en el nivel de satisfacción, de los turistas extranjeros, y la intención de recomendación de los turistas que visitan la ciudad del Cusco, utilizando métodos multivariantes como el Modelo de Regresión Logística Múltiple [7]. Los datos usados corresponden a la encuesta realizada por la Corporación de Compañías de Research (CCR), por encargo de PROMPERÚ, en el año 2003.

Palabras claves: Análisis multivariante, Análisis de Regresión Logística Múltiple, Turismo, Wald, Odds Ratio.

\begin{abstract}
In the present work, we determine the most important factors in the nivel of satisfaction of foreign tourists, and the intention of recommendation of the tourists who visit the city of the Cusco using multivariate methods such as the Multiple Logistic Regression Model [7]. The data used correspond to the survey conducted by the company Corporación de Compañias de Research CCR, on behalf of PROMPERÚ, in 2003.
\end{abstract}

Key words: Multivariate analysis, Multiple Logistic Regression, Tourism, Wald, Odds Ratio.

\section{INTRODUCCION}

El turismo es una actividad con un creciente desarrollo, representa alrededor del $4 \%$ del PBI y gracias a esfuerzos continuos, el Perú goza ahora de una imagen turística importante en el mercado internacional. Las actividades que se vienen desarrollando para mejorar el desempeño de los operadores turísticos y otros relacionados, poco a poco van teniendo mejores resultados.

\footnotetext{
${ }^{1}$ CCR, Corporación de Compañías de Research, Lima, Perú.

${ }^{2}$ UNMSMS, Facultad de Ciencias Matemáticas, Lima - Perú.
} 
Como ente estatal encargado de esta labor, PROMPERÚ tiene como misión liderar la promoción del producto turístico peruano y la imagen del país, tanto en el ámbito nacional como en el internacional; en este contexto, realiza diversas actividades y recurre a múltiples fuentes; así, además de contar con indicadores del desenvolvimiento del sector: llámese entradas de extranjeros, capacidad hotelera instalada, centros de Información turística, guías, etc, utiliza la investigación de mercado con el fin de conocer la percepción del cliente; esto es, del turista. Una de las variables relevantes a conocer es el nivel de satisfacción del turista que visita el Perú, tanto el turista interno como el turista externo. Este último es de particular interés porque representa una fuente de divisas importante, con lo cual el mercado potencial de turistas tendería a crecer.

Saber cómo se siente el turista puede determinarse por qué tan satisfecho estuvo con la atención recibida en los diferentes ámbitos: capacidad hotelera, transporte, seguridad y orden del lugar visitado, entre otras razones más. Para ello, la empresa de investigación de mercados CCR por encargo de PROMPERU desarrolló en el año 2003 el estudio para determinar el nivel de satisfacción del turista extranjero que visita el Perú El análisis estadístico se realizó solamente con métodos estadísticos univariantes [11].

En ese contexto, el objetivo del presente trabajo es determinar los factores más importantes en el nivel de satisfacción del turista extranjero, que visita la ciudad del Cusco, y también modelar cómo influye en la intención de recomendación, mediante el uso de métodos multivariantes como el Modelo de Regresión Logística Múltiple. A partir de la información obtenida por PROMPERU [11].

\section{METODOLOGÍA ESTADÍSTICA}

\subsection{MODELO DE REGRESIÓN LOGÍSTICA MÚLTIPLE}

El Modelo de regresión logística es una de las herramientas estadísticas con mejor capacidad para el análisis de datos, en particular en investigación clínica y epidemiológica, aunque en general las aplicaciones son diversas e incluyen las áreas de negocios y finanzas, criminología, ecología, ingeniería, biología y otros, lo que se verifica en el presente trabajo.

La regresión logística permite modelar, cómo influye en la probabilidad de aparición de un suceso, habitualmente dicotómico, la presencia o no de diversos factores y el valor o nivel de los mismos. También puede usarse para modelar la probabilidad de aparición 
de cada una de las posibilidades de un suceso con más de dos categorías (politómico ó multinomial).

El modelo de regresión lineal exige una serie de suposiciones que usualmente son difíciles de cumplir (variables continuas, linealidad de las variables por ejemplo) [4]. La ventaja del análisis de regresión logística es que no hay ninguna suposición en cuanto a la distribución de la probabilidad de las variables explicativas, por lo que pueden intervenir variables explicativas no normales y variables cualitativas. Si se tiene dos grupos, de tal manera que un sujeto pertenece al grupo 1 o 2 (está satisfecho con la visita al Perú o no lo está), el modelo de regresión logística permite estimar la probabilidad de pertenecer a uno de esos grupos. Cuando se tiene más de dos grupos, el modelo se puede extender de forma natural, con pocas modificaciones, conociéndose entonces como regresión logística politómica [7].

Muchas funciones de distribución han sido propuestas para ser usadas en el análisis de una variable respuesta dicotómica o politómica [7]. La razón por la que se elige en el presente trabajo es por ser un modelo extremadamente flexible y fácil de usar, lo que facilita las correspondientes interpretaciones.

A continuación se presenta el modelo de regresión logística múltiple. Para un conjunto de $k$ variables independientes representadas en el vector $x^{\prime}=\left(x_{1}, x_{2}, \ldots, x_{k}\right)$, denotemos por:

$$
P(Y=1 / x)=\pi(x)
$$

la probabilidad condicional que la respuesta esté presente,

$$
g(x)=\beta_{0}+\beta_{1} x_{1}+\beta_{2} x_{2}+\ldots+\beta_{k} x_{k}
$$

el logit del modelo de regresión logística múltiple, donde $\beta_{0}, \beta_{1}, \beta_{2}, \ldots, \beta_{k}$ son los parámetros del modelo de regresión logística múltiple y

$$
\pi(x)=\frac{e^{g(x)}}{1+e^{g(x)}}
$$

es el modelo de regresión logística múltiple [4].

En el modelo (2.2) se tienen que estimar los parámetros y evaluar la adecuación del modelo, por lo que a continuación se hace una breve presentación de dichos aspectos. 
La variable respuesta, Y, puede ser codificada por 0 ó 1, representando la ausencia o la presencia de la característica de interés.

\subsection{ESTIMACIÓN DE LOS PARÁMETROS}

Mediante una muestra de $n$ observaciones independientes $\left(x_{i}, y_{i}\right), i=1,2, \ldots, n$, donde $x_{i}$ es el valor de la variable independiente para el i-ésimo individuo e $y_{i}$ denota el valor de la variable respuesta dicotómica, se obtienen los estimadores del vector de parámetros, $\beta^{\prime}=\left(\beta_{0}, \beta_{1}\right), \ldots, \beta_{k}$, mediante el método de máxima verosimilitud [7].

La función de máxima verosimilitud es

$$
l(\beta)=\prod_{i=1}^{n} \pi\left(x_{i}\right)^{y_{i}}\left[1-\pi\left(x_{i}\right)\right]^{1-y_{i}}
$$

donde $\pi(x)$ se define como en la ecuación (2.2) para cada $x_{i}$. La aplicación del método de máxima verosimilitud conlleva a $k+1$ ecuaciones de verosimilitud que se obtienen por diferenciación de la función logaritmo de la verosimilitud con respecto a los $k+1$ parámetros. Las ecuaciones de verosimilitud que resultan se expresan de la siguiente manera:

$$
\sum_{i=1}^{n}\left[y_{i}-\pi\left(x_{i}\right)\right]=0 \text { y } \sum_{i=1}^{n} x_{i j}\left(y_{i}-\pi\left(x_{i}\right)\right), \text { para } j=1,2, \ldots, p
$$

La solución de estos conjuntos de ecuaciones mediante métodos iterativos permite estimar los parámetros del modelo, para los que existen softwares estadísticos tales como el SPSS, MINITAB, STATA, entre otros.

\subsection{EVALUACIÓN DEL MODELO}

\subsubsection{ESTADÍSTICA G}

Una vez que se ha ajustado un modelo de regresión logística multivariante es conveniente realizar el proceso de evaluación del mismo, con la finalidad de saber si las variables independientes explican bien el comportamiento de la variable dependiente.

Es decir, se evalúa la significancia del modelo, comparando el modelo con todas las variables explicativas frente al modelo sin ninguna variable explicativa. Es decir, el test estadístico para decimar la hipótesis nula $H_{0}$ frente a la hipótesis alternativa $H_{1}$, 
$H_{0}: \beta_{1}=\beta_{2}=\ldots=\beta_{k}=0$

$H_{1}: \beta_{i} \neq 0$ para algún $\beta_{i}$

se basa en la estadística $G$ :

$$
\begin{gathered}
G=D \text { (modelo sin las variables) }-D \text { (modelo con las variables) } \\
G=2\left\{\sum_{i=1}^{n}\left[y_{i} \ln \left(\widehat{\pi}_{i}\right)+\left(1-y_{i}\right) \ln \left(1-\widehat{\pi}_{i}\right)\right]-\left[n_{1} \ln \left(n_{1}\right)+n_{0} \ln \left(n_{0}\right)-n \ln (n)\right]\right\}
\end{gathered}
$$

Bajo la hipótesis nula, que postula que todos los $k$ parámetros son iguales a cero, la estadística $G$ tiene una distribución $C h i$-cuadrado con $k$ grados de libertad. Es decir, $G \sim X^{2}$ con $k$ grados de libertad.

Cuando se rechaza la hipótesis nula, se concluye que por lo menos uno de los $k$ coeficientes son diferentes de cero, una interpretación análoga al de regresión lineal múltiple. Es decir, si $G>X_{\alpha, k}^{2}$ rechazamos $H_{0}$, y concluimos que al menos uno de los coeficientes es diferente de cero; donde $X_{\alpha, \beta}^{2}$ es el cuantil de la distribución Chi-cuadrado.

Decisión: Si $W>X_{\alpha, k}^{2}$, se rechaza la hipótesis nula, $H_{0}$, con un nivel de significancia fijado $\alpha$, y concluimos que al menos una de las variables independientes son diferentes de cero.

Una vez encontrado el mejor conjunto de variables explicativas que predicen la variable $Y$, se debe evaluar cada coeficiente para determinar cuál o cuáles de las variables ingresan al modelo, para lo cual se utiliza la estadística de Wald.

\subsubsection{ESTADÍSTICA DE WALD}

Se evalúa la significancia de cada uno de los parámetros mediante la comparación de las hipótesis nula, $H_{0}$, y alternativa, $H_{1}$,

$H_{0}: \beta_{i}=0 ; i=1,2, \ldots, k$

$H_{1}: \beta_{i} \neq 0$ para algún $\beta_{i}$

La estadística de prueba es:

$$
W=\widehat{\beta}^{\prime}[V \widehat{a} r \widehat{\beta}]^{-1} \widehat{\beta}=\widehat{\beta}^{\prime}\left(X^{\prime} V X\right) \widehat{\beta}
$$

donde $V$ es una matriz diagonal de dimensión $n \times n$, donde 


$$
V=\left[\begin{array}{cccc}
\widehat{\pi}_{1}\left(1-\widehat{\pi}_{1}\right) & 0 & \ldots & 0 \\
0 & \widehat{\pi}_{2}\left(1-\widehat{\pi}_{2}\right) & \ldots & 0 \\
\vdots & 0 & \ddots & \vdots \\
0 & \ldots & \ldots & \widehat{\pi}_{n}\left(1-\widehat{\pi}_{n}\right)
\end{array}\right]
$$

$X$ es una matriz de dimensión $n \times(k+1)$, donde

$$
X=\left[\begin{array}{ccccc}
1 & x_{11} & x_{12} & \ldots & x_{1 k} \\
1 & x_{21} & x_{22} & \ldots & x_{2 k} \\
1 & \vdots & \vdots & \vdots & \vdots \\
1 & x_{n 1} & x_{n 2} & \ldots & x_{n k}
\end{array}\right]
$$

Bajo la hipótesis nula de que cada uno de los $k+1$ coeficientes son iguales a cero, la estadística $W$ tiene una distribución Chi-cuadrado con $k+1$ grados de libertad. Es decir, $W \sim X_{\alpha, k+1}^{2}$.

Decisión: Si $W>X_{\alpha, k}^{2}$ se rechaza la hipótesis nula, $H_{0}$, con un nivel de significancia fijado $\alpha$, y concluimos que al menos uno de los parámetros pueden ser diferente de cero.

\subsubsection{PRUEBA DE HOSMER - LEMESHOW}

Evalúa la bondad de ajuste del modelo construyendo una tabla de contingencia. Divide la muestra en aproximadamente 10 grupos iguales a partir de las probabilidades estimadas, para comparar las frecuencias observadas con las esperadas en cada uno de estos grupos a través de la prueba $X^{2}$ con $j-2$ grados de libertad, en donde $j$ es el número de grupos formados [7].

Se calculan los deciles de las probabilidades estimadas $\widehat{\pi}_{i} ; i=1, \ldots, n$ y $D_{1}, \ldots, D_{9}$ que son los deciles observados divididos en 10 grupos dados por:

$A_{j}=\left\{i \in\{1, \ldots, n\} ; \widehat{\pi}_{i} \in\left[D_{j-1}, D_{j}\right]\right\}, j=1, \ldots, 10$

donde: $D_{0}=0, D_{10}=1$

Sean:

$n_{j}$ : número de casos en $A_{j}, j=1, \ldots, 10$

$o_{j}$ número de $y_{i}=1$ en $A_{j}, j=1, \ldots, 10$

$\bar{\pi}_{j}=\frac{1}{n} \sum_{i \in A_{j}} \widehat{\pi}_{i} ; j=1, \ldots, 10$

Las hipótesis a contrastar son:

$H_{0}:$ El modelo es adecuado 
$H_{1}$ : El modelo es inadecuado

La estadística de prueba es:

$$
x^{2}=\sum_{j=1}^{10} \frac{\left(o_{j}-n_{j} \bar{\pi}_{j}\right)^{2}}{\bar{\pi}_{j} n_{j}\left(1-\bar{\pi}_{j}\right)} \sim X_{\alpha, j-2}^{2}
$$

Decisión: Si $X^{2}>X_{\alpha, j-2}^{2}$ se rechaza $H_{0}$ y se concluye que el modelo no es adecuado a un nivel de significancia $\alpha$.

\section{DESCRIPCIÓN DE LOS DATOS}

Los datos utilizados en la presente investigación fueron tomados de PROMPERU [8]. Uno de los objetivos de Promperú fue determinar el grado de satisfacción de los turistas con respecto a los servicios turísticos utilizados durante su viaje, para lo cual emplearon una escala de cinco categorías, $5=$ Totalmente insatisfecho, $4=$ Insatisfecho, $3=\mathrm{Ni}$ satisfecho ni insatisfecho, 2 = Satisfecho y 1 = Totalmente satisfecho. Con esta escala se solicitó calificar el servicio turístico y cada uno de los atributos propuestos que caracterizan el servicio. Midieron también "intención de recomendar la ciudad como destino turístico", variable que más allá de demostrar satisfacción, indica real impacto en la visita, debido a que estarían dispuestos a recomendar a sus familiares o amigos el Perú como destino turístico. La escala utilizada para medir la intención de recomendación fue; $1=$ Los desanimaría, 2 = Ni les recomendaría ni los desanimaría, $3=$ Recomendaría, pero con reservas, 4 = Recomendaría sin ninguna duda que visiten la ciudad.

La población en estudio fueron turistas hombres y mujeres mayores de 15 años, de nacionalidad y residencia extranjera procedentes de Norteamérica, Sudamérica y Europa, que visitaron la ciudad del Cusco y permanecieron por lo menos dos noches en la ciudad.

La aplicación del cuestionario fueron realizadas en los principales puntos de salida de la ciudad de Cusco. Aeropuerto Alejandro Velasco Astete, Terminal de transporte terrestre y Estación del tren Cusco.

Se consideró una muestra aleatoria de 292 turistas (máximo margen de error de $\pm 5.7 \%$ al $95 \%$ de nivel de confianza) que visitaron el Cusco por vacaciones, recreación u ocio.

Las variables consideradas en el presente estudio se encuentran en la Tabla de variables. 


\begin{tabular}{|c|c|c|}
\hline Variables & Codificación & Nombre \\
\hline $\begin{array}{l}\text { 1. Satisfacción con los } \\
\text { establecimientos de hospedaje }\end{array}$ & $\begin{array}{l}\text { 0. Regularmente o no } \\
\text { satisfecho } \\
\text { 1. Satisfecho } \\
\text { 2. Muy satisfecho }\end{array}$ & HOSPED \\
\hline $\begin{array}{l}\text { 2. Satisfacción con el servicio de } \\
\text { transporte aéreo. }\end{array}$ & $\begin{array}{l}\text { 0. Regularmente o no } \\
\text { satisfecho } \\
\text { 1. Satisfecho } \\
\text { 2. Muy satisfecho }\end{array}$ & AEREO \\
\hline $\begin{array}{l}\text { 3. Percepción de seguridad en la } \\
\text { ciudad }\end{array}$ & $\begin{array}{l}\text { 0. Poco o nada segura } \\
\text { 1. Segura } \\
\text { 1. Muy segura }\end{array}$ & SEGUR \\
\hline $\begin{array}{l}\text { 4. Percepción del orden del } \\
\text { tránsito }\end{array}$ & $\begin{array}{l}\text { 0. Poco o nada ordenado } \\
\text { 1. Ordenado } \\
\text { 2. Muy ordenado }\end{array}$ & TRANS \\
\hline 5. Vez que visita el Perú & $\begin{array}{l}0 \text { Primera vez } \\
1 \text { No es la primera vez }\end{array}$ & VISITA \\
\hline 6. Edad & Variable continua & EDAD \\
\hline 7. Sexo & $\begin{array}{l}0 \text { Masculino } \\
1 \text { Femenino }\end{array}$ & SEXO \\
\hline 8. Continente & $\begin{array}{l}0 \text { Norteamérica } \\
1 \text { Europa } \\
2 \text { Sudamérica }\end{array}$ & CONTIN \\
\hline $\begin{array}{l}\text { 9. Varianle respuesta: } \\
\text { Intención de recomendación }\end{array}$ & $\begin{array}{l}0 \text { Recomendaría con reservas } \\
\text { o no recomendaría } \\
1 \text { Recomendaría sin ninguna } \\
\text { duda }\end{array}$ & $\mathrm{REC}$ \\
\hline
\end{tabular}

Tabla de Variables

\section{RESULTADOS Y DISCUSIÓN}

Dado el número de variables y número de turistas que conformaron la muestra, para aplicar a los datos obtenidos la metodología planteada en (2) se utilizó la versión 13 del programa estadístico Statistical Package off Social Sciences (SPSS).

Luego de hacer el ajuste de diversos modelos, se eligió el mejor de ellos representado por las variables que se presentan en la Tabla 1.

La Tabla 1 muestra las variables significativas del modelo ajustado. Podemos concluir que los factores de influencia en la intención de recomendación de visitar Cusco son: Edad $(\mathrm{p}=0,019)$, Hospedaje $(\operatorname{Hosped}(1)$ y Hosped $(2)(\mathrm{p}=0,00)) ;$ y Percepción de Seguridad en la ciudad $(\operatorname{Segur}(1)$ y $\operatorname{Segur}(2)(\mathrm{p}=0,00))$, a un nivel de significación del 5\%. 


\begin{tabular}{|c|c|c|c|c|c|c|}
\hline Variables & $\hat{\beta}$ & $\begin{array}{c}\text { Error } \\
\text { estándar }\end{array}$ & Wald & $\begin{array}{c}\text { Grados de } \\
\text { libertad }\end{array}$ & Sig. & $\operatorname{Exp}(\hat{\beta})$ \\
\hline Edad & $-0,025$ & 0,010 & 5,540 & 1 & 0,019 & 0,976 \\
\hline Hosped & & & 20,269 & 2 & 0,000 & \\
\hline Hosped(1) & 1,487 & 0,389 & 14,586 & 1 & 0,000 & 4,423 \\
\hline Hosped(2) & 2,016 & 0,517 & 15,210 & 1 & 0,000 & 7,505 \\
\hline Segur & & & 16,727 & 2 & 0,000 & \\
\hline Segur(1) & 1,556 & 0,419 & 13,780 & 1 & 0,000 & 4,738 \\
\hline Segur(2) & 2,392 & 0,734 & 10,612 & 1 & 0,001 & 10,936 \\
\hline
\end{tabular}

Tabla 1: Resultados del SPSS para el Modelo a ajustar.

Con los valores de la columna donde se presenta las estimaciones de los parámetros, se presenta el modelo ajustado, que estima el modelo (2.2). El modelo ajustado para identificar los factores mas influyentes en la intención de recomendación de visitar Cusco es el siguiente:

$$
p_{i}=\frac{1}{1+e^{-z}}
$$

donde,

$z=-0.025$ Edad $+1,487$ Hosped $(1)+2,016$ Hosped $(2)+1,556$ Segur $(1)+2,392$ Segur $(2)$

Para evaluar la bondad de ajuste del modelo utilizamos la prueba de Hosmer-Lemeshow, cuyos resultados se presentan en la Tabla 2 , la que muestra que el ajuste es bueno $(\mathrm{p}=0,33)$ a un nivel de significancia del $5 \%$. Luego, los datos se ajustan al modelo de regresión logística múltiple.

\begin{tabular}{|c|c|c|}
\hline $\begin{array}{c}\text { Chi } \\
\text { cuadrado }\end{array}$ & $\begin{array}{c}\text { Grados de } \\
\text { libertad }\end{array}$ & Sig. \\
\hline 9,144 & 8 & 0,33 \\
\hline
\end{tabular}

Tabla 2: Prueba de Hosmer y Lemeshow.

A partir de los resultados se puede decir que, en cuanto a la percepción de la seguridad en la ciudad: existen aproximadamente 5 veces más posibilidades que el turista recomiende sin reservas a visitar nuestro país, si la ciudad es percibida por él como segura, respecto a si no fuera segura. $\left(e^{1,556}=4,74, O R=4,74>1\right)$. 
Asimismo, existen aproximadamente 11 veces más posibilidades que el turista recomiende sin reservas visitar nuestro país si la ciudad es percibida como muy segura, respecto a si no fuese segura. $\left(e^{2,392}=10,94, O R=10,94>1\right)$

En lo referente a la variable hospedaje, existen aproximadamente 4 veces más posibilidades que el turista recomiende sin reservas visitar nuestro país, si el hospedaje en el que se alojó generó satisfacción. $\left(e^{1,487}, O R=4,4>1\right)$. Existen aproximadamente 8 veces más posibilidades que el turista recomiende sin reservas visitar nuestro país si el hospedaje en el que se alojó generó mucha satisfacción $\left(e^{2,016}, O R=7,5>1\right)$.

Para una mejor interpretación de la variable edad más, se toma la diferencia de la edad en el lapso de 10 años. Así,

$$
\begin{aligned}
& \operatorname{Logit}\left(\hat{u}_{\text {est }} / \hat{u}=50\right)=-0,025(50)=-1,25 \\
& \operatorname{Logit}\left(\hat{u}_{\text {est }} / \hat{u}=40\right)=-0,025(40)=-1 \\
& \operatorname{Logit}\left(\hat{u}_{\text {est }} / \hat{u}=50\right)-\operatorname{Logit}\left(\hat{u}_{\text {est }} / \hat{u}=40\right)=-1,25-(-1)=-0,25 .
\end{aligned}
$$

De donde, la posibilidad de que un turista recomiende sin dudas visitar nuestro país, es sólo el $78 \%$ en un turista de 50 años, respecto a uno de 40 años $\left(O R=e^{-0,25}=0,78\right)$. La intención de recomendar nuestro país, desciende conforme se incrementa la edad del

\begin{tabular}{|c|c|c|c|}
\hline \multirow[b]{2}{*}{$\begin{array}{c}\text { Recomendación } \\
\text { observada }\end{array}$} & \multicolumn{2}{|c|}{ Recomendación estimada } & \multirow[b]{2}{*}{$\begin{array}{c}\text { Porcentaje } \\
\text { Correcto }\end{array}$} \\
\hline & $\begin{array}{l}\text { Con reservas o } \\
\text { no lo haría }(0)\end{array}$ & $\begin{array}{l}\text { Sin duda } \\
\text { alguna (1) }\end{array}$ & \\
\hline $\begin{array}{l}\text { Con reservas o } \\
\text { no lo haría }(0)\end{array}$ & & & Especificidad \\
\hline $\begin{array}{l}\text { Sin duda } \\
\text { alguna (1) }\end{array}$ & & & Sensibilidad \\
\hline
\end{tabular}
turista.

Sea una tabla de clasificación

Sea $\mathrm{B}=(\mathrm{RECOM}=0) /(\mathrm{RECOM}=0)$ ESPECIFICIDAD DE LA ECUACION DE PRONOSTICO

Sea $\mathrm{A}=(\mathrm{RECOM}=1) /(\mathrm{RECOM}=1)$ SENSIBILIDAD DE LA ECUACION DE PRONOSTICO

Como el paquete estadístico SPSS proporciona el valor de corte en 0,5 como predeterminado, se ha corrido el modelo para los valores desde 0,1 hasta 0,9 cada 0,05 , como se resume a continuación. 


\begin{tabular}{|c|c|c|c|}
\hline $\begin{array}{c}\text { Punto de } \\
\text { corte }\end{array}$ & Especificidad & Sensibilidad & Total \\
\hline 0.05 & 0.00 & 100.00 & 85.57 \\
\hline 0.10 & 0.00 & 100.00 & 85.57 \\
\hline 0.15 & 0.00 & 100.00 & 85.57 \\
\hline 0.20 & 2.38 & 100.00 & 85.91 \\
\hline 0.25 & 2.38 & 100.00 & 85.91 \\
\hline 0.30 & 2.38 & 100.00 & 85.91 \\
\hline 0.35 & 7.14 & 100.00 & 86.60 \\
\hline 0.40 & 11.90 & 99.60 & 86.94 \\
\hline 0.45 & 11.90 & 99.60 & 86.94 \\
\hline 0.50 & 11.90 & 99.20 & 86.60 \\
\hline 0.55 & 21.43 & 98.80 & 87.63 \\
\hline 0.60 & 23.81 & 97.19 & 86.60 \\
\hline 0.65 & 26.19 & 95.98 & 85.91 \\
\hline 0.70 & 33.33 & 89.96 & 81.79 \\
\hline 0.75 & 45.24 & 85.94 & 80.07 \\
\hline 0.80 & 52.38 & 83.94 & 79.38 \\
\hline 0.85 & 61.90 & 78.71 & 76.29 \\
\hline 0.90 & 73.81 & 62.65 & 64.26 \\
\hline
\end{tabular}

Se debe elegir un punto de corte óptimo que maximice tanto la sensibilidad como la especificidad, esto es más fácil de observar en el siguiente gráfico, donde el punto de corte óptimo lo identificamos como 0,87 .

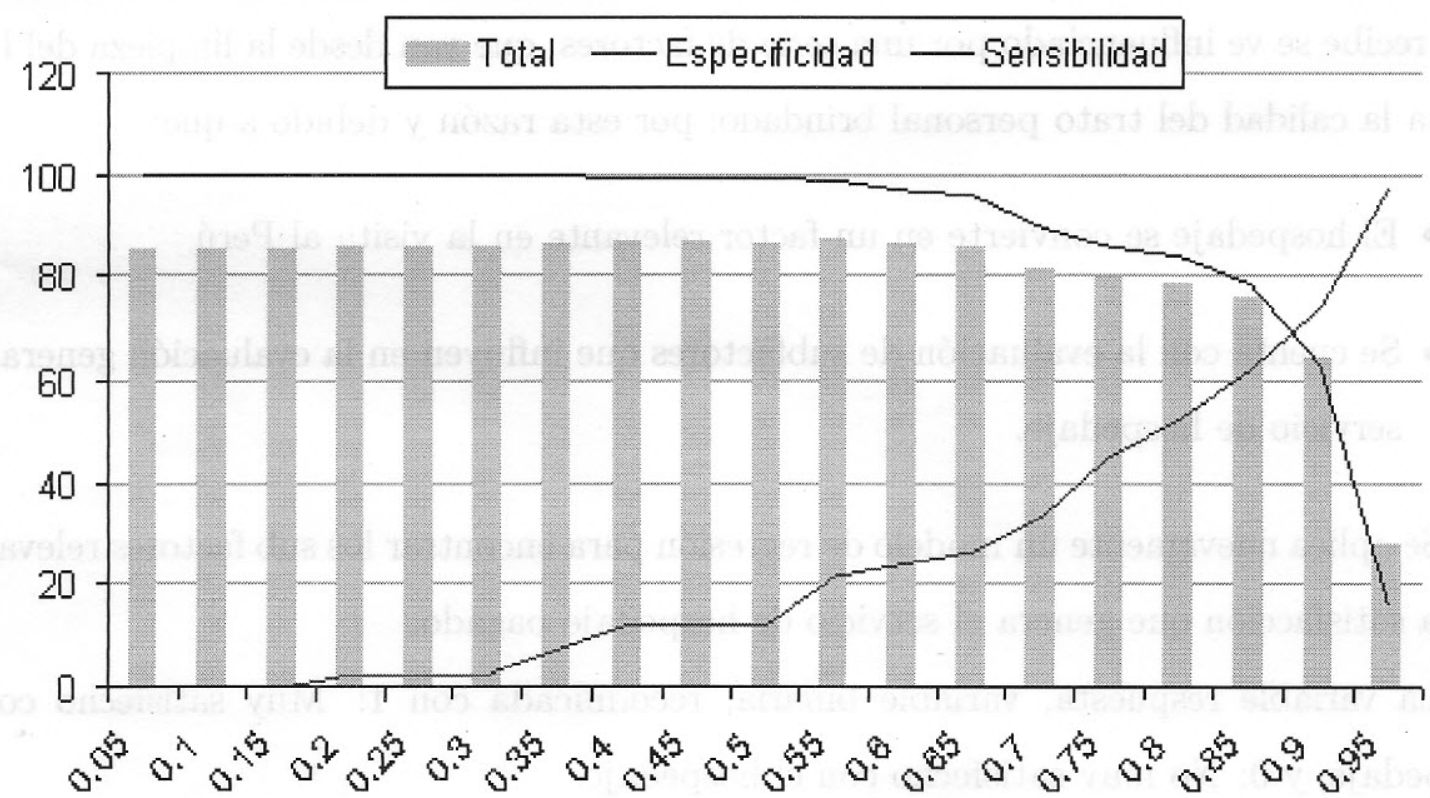

Gráf. 1: Evolución de la curva de ROC.

En la Tabla 3 presentamos la tabla de clasificación de los turistas según el modelo 
propuesto, según el modelo se predice correctamente la recomendación del Cusco como destino turístico en un $76,3 \%$ de los casos. Por el contrario, la no recomendación es correctamente clasificada en un 66,7\%. La Tabla de clasificación muestra que, con el modelo, se clasifica correctamente la intención de recomendación de la ciudad del Cusco, en un $74,9 \%$.

\begin{tabular}{|c|c|c|c|c|}
\hline \multirow{2}{*}{$\begin{array}{c}\text { Intención de } \\
\text { Recomendación } \\
\text { observada }\end{array}$} & \multicolumn{2}{|c|}{$\begin{array}{c}\text { Intención de Recomendación } \\
\text { estimada }\end{array}$} & \multirow{2}{*}{\multicolumn{2}{|c|}{ Porcentaje Correcto }} \\
\hline & $\begin{array}{c}\text { Con reservas o } \\
\text { no lo haría }\end{array}$ & Sin duda alguna & & \\
\hline $\begin{array}{c}\text { Con reservas o } \\
\text { no lo haría }\end{array}$ & 28 & 14 & & 66,7 \\
\hline Sin duda alguna & 59 & 190 & & 76,3 \\
\hline
\end{tabular}

Porcentaje correcto global de clasificación $74,9 \%$

Tabla 3: Tabla de clasificación para el modelo propuesto.

Para saber que factores son relevantes en el nivel de satisfacción del turista extranjero con respecto al hospedaje utilizado, se uso esta variable, a continuación mostramos los factores considerados en este rubro.

\section{Construcción del modelo para la variable hospedaje}

Cuando un turista o en general un cliente se hospeda en un establecimiento, el servicio que recibe se ve influenciado por una serie de factores, que van desde la limpieza del lugar hasta la calidad del trato personal brindado; por esta razón y debido a que:

- El hospedaje se convierte en un factor relevante en la visita al Perú

- Se cuenta con la evaluación de subfactores que influyen en la evaluación general del servicio de hospedaje.

Se aplica nuevamente un modelo de regresión para encontrar los sub factores relevantes en la satisfacción que genera el servicio de hospedaje pagado.

La variable respuesta, variable binaria; recodificada con 1: Muy satisfecho con el hospedaje, y 0: No muy satisfecho con el hospedaje.

Los sub factores o variables explicativas son:

- Limpieza del hospedaje 
- Trato personal recibido

- Comodidad del hospedaje

- Seguridad en el hospedaje

- Precio del hospedaje

- Infraestructura del hospedaje

- Ubicación del hospedaje

Todas estas variables han sido recodificadas, como 0: No muy satisfecho, y 1: Muy satisfecho.

El modelo de regresión logística binaria construido considera siete variables independientes, todas categóricas.

Finalmente, los coeficientes para las variables significativas están dadas en la Tabla 4.

\begin{tabular}{|c|c|c|c|c|c|c|}
\hline Variables & $\hat{\beta}$ & $\begin{array}{c}\text { Error } \\
\text { estándar }\end{array}$ & Wald & $\begin{array}{c}\text { Grados de } \\
\text { libertad }\end{array}$ & $\operatorname{Sig.}$ & $\operatorname{Exp}(\hat{\beta})$ \\
\hline Limpieza(1) & 1,270 & 0,448 & 8,019 & 1 & 0,005 & 3,561 \\
\hline Tra_pers(1) & 2,123 & 0,510 & 17,306 & 1 & 0,000 & 8,354 \\
\hline Comodi(1) & 1,676 & 0,430 & 15,218 & 1 & 0,000 & 5,345 \\
\hline Segurid(1) & 1,395 & 0,434 & 10,346 & 1 & 0,001 & 4,035 \\
\hline Infraest(1) & 1,058 & 0,419 & 6,390 & 1 & 0,011 & 2,882 \\
\hline Constante & $-4,852$ & 0,653 & 55,192 & 1 & 0,000 & 0,008 \\
\hline
\end{tabular}

Tabla 4 : Variables en el Modelo.

Los subfactores de mayor influencia en la satisfacción total del servicio de Hospedaje son : Trato personal $(p=0,000)$; Comodidad de la habitación $(p=0,000)$; Seguridad en el establecimiento ( $p=0,001)$; Limpieza del lugar $(p=0,005)$; Infraestructura del hospedaje ( $p=0,011)$, a un nivel de significancia del $5 \%$.

La satisfacción total con el hospedaje es ocho veces mayor en un cliente que está muy satisfecho con el trato personal recibido, frente a un cliente que no tiene ese nivel de satisfacción.

La comodidad de la habitación en el hospedaje es el segundo subfactor en relevancia y eleva cinco veces la posibilidad de lograr satisfacción total. 


\section{CONCLUSIONES}

Los factores identificados como los de mayor relevancia en la satisfacción total del turista son el alojamiento pagado y la seguridad en la ciudad.

El alojamiento pagado, se convierte así en un servicio clave para la satisfacción del turista. Particularmente influyen en este servicio, el trato personal que se brinda al cliente, la comodidad y seguridad tanto en la habitación como en el establecimiento, la infraestructura y limpieza del alojamiento. El precio y la ubicación no influirían de modo relevante en la satisfacción con este servicio.

En cuanto a la seguridad en la ciudad: un turista que percibe a la ciudad del Cusco como un sitio muy seguro recomendaría la ciudad como destino turístico 10 veces más respecto de uno que no la percibió segura. En este tema juegan un rol importante las recomendaciones de terceros, pues si bien la mayoría de turistas no se ha visto afectado, de manera directa, por un problema de inseguridad, lo que "escucha" y las "recomendaciones de terceros" originan en él desconfianza y temor. No se debe descuidar la presencia policial y vigilancia de serenazgo, así como una capacitación interna a los que interactúan con los turistas de modo tal que si bien el turista sea advertido de los lugares o puntos en los que debe tener cuidado, no se llegue a la "alarma" innecesaria.

La edad del turista tiene influencia en la intención de recomendación, así la recomendación se incrementaría según el turista sea más joven; ello se explicaría en que el Perú, y Cusco en particular, es clasificado de modo importante como un destino - además de histórico y cultural - de naturaleza y aventura; nuestra rica geografía y enorme patrimonio cultural, nos permiten brindar al mundo un atractivo completo.

Aunque el Orden del Tránsito no ha sido identificado como un factor que determine o influya de modo negativo en la intención de recomendar la visita a nuestro país, se debe tener en cuenta el notorio desorden de nuestro sistema vehicular, Cusco soporta actualmente más de tres mil taxis además de centenares de buses turistas que circulan en calles que fueron diseñadas para el tráfico de mulas, por lo que, en aras de la preservación de los monumentos históricos, de la propia ciudad y de la tranquilidad del visitante (ruidos molestos ocasionados por el uso del claxon), se requiere inmediata atención en este tema.

El transporte aéreo utilizado no se torna claramente como un factor de influencia negativo, pero demás está decir que contar con buen número de agencias aéreas calificadas 
implica la percepción de un país mejor preparado para el turismo receptivo y con mejor soporte logístico y físico. En particular se debe prestar especial atención al estado del avión.

No influye en la satisfacción total del turista el que éste haya venido una o más veces, la intención de recomendación es la misma, ya sea que el turista viene por vez primera, o ya lo ha hecho antes.

Además de la consideración de estas variables; es importante atender otras falencias como son la contaminación, el acoso de los vendedores, el precio elevado de algunos productos y servicios y en general la percepción de pobreza observada por el turista en su paso por nuestro país. 


\section{REFERENCIAS}

[1] ANDERSEN, E.B. Introduction to the statistical analysis of categorical. SpringerVerlag Berlin Heidelberg. New York, 1997.

[2] ALVAREZ, C.R. Estadística multivariante y no paramétrica con SPSS: Aplicación a las ciencias de la salud. Díaz de Santos, S.A. Madrid, 1995.

[3] ATKINSON, A.C. Plots, transformations, and regression: An Introduction to graphical methods of diagnostic regression analysis. New York : Clarendon Press Oxford. 1985. 282p.

[4] CASTRO, J.; PURIFICACIÓN, G. Estadística Multivariante. Amaru ediciones y autores. España, 2000.

[5] CCR, Corporación de Compañías de Research, Lima, Perú. Fuente de los datos del Nivel de Satisfacción del Turista Extranjero 2003. Lima, Perú, 2003.

[6] DOMINGUEZ, C.R.. Factores que influyen en el nivel de satisfacción de los turistas extranjeros que visitan cusco. 68f. Monografía (Licenciada en Estadística) - Facultad de Ciencias Matemáticas, Universidad Nacional Mayor de San Marcos, Lima, Perú, 2005.

[7] HOSMER, D.W.; LEMESHOW, S. Applied logistic regression. 2nd. ed. John Wiley \& Sons. New York, 2000.

[8] LONG, J.S. Regression models for categorical and limited dependent variables. Sage Publications. California, 1997.

[9] PEREZ, C. Técnicas estadísticas con spss. Prentice Hall, Madrid, 2001.

[10] PREGIBON, D. Logistic regression diagnostics. Ann. Stat., v.40, p.705-724, 1980.

[11] PROMPERU. Nivel de Satisfacción del Turista Extranjero 2003. Lima, Perú, 2003.

[12] SOLANO, O.; BARTOLO, F.; GIRALDO, O.; SAlINAS, S. Análisis de diagnóstico en el modelo de regresión logística para determinar los factores de riesgo en la osteoporosis. Informe final presentado al Instituto de Investigación de la Facultad de Ciencias Matemáticas: UNMSM, Lima. Perú, 2004.

[13] VISAUTA, B. Análisis estadístico con spss para windows: Estadística Multivariante. McGraw-Hill, Madrid, 1998.

[14] WEISBERG, S. Applied linear regression. 2nd. ed. John Wiley \& Sons. New York, 1985. 